\title{
Local differences in electrogastrographic responses to the stress of the mirror drawing test (MDT) as determined by multichannel electrogastrography
}

\author{
Shinji Номма ${ }^{1}$ \\ ${ }^{1}$ Division of Organ Physiology, Department of Regenerative and Transplant Medicine, \\ Institute of Medicine and Dentistry, Niigata University, Japan
}

Received March 29, 2012; Accepted May 10, 2012

\begin{abstract}
Electrogastrograms (EGGs) were recorded in human subjects $(\mathrm{n}=58)$ at 16 locations on the thoraco-abdominal surface before (at rest) and during the stress of the mirror drawing test (MDT) and after having a test meal. The power amplitude ratios and power content ratios of MDT to rest (MDT/rest) and postprandial state to rest (PPR/rest) were compared between gastric channels 5 and 8 , and the infraumbilical channels $12,13,14,15$, and 16. Generally, the ratio of the 3 -cpm group in channels 5 and 8 was lower than that of the infraumbilical channels 12-16 during MDT. In contrast, the ratios were higher in the epigastric channels than in the infraumbilical channels after a test meal. Significant differences between the epigstric and infraumbilical channels were found in the comparisons of the power content ratios. The infraumbilical channels were facilitated and the epigastric channels inhibited during MDT. After eating a meal, in contrast, the postprandial epigastric 3-cpm EGGs were facilitated. The epigastric 3-cpm EGG activity reflects gastric myoelectric activity, while the infraumbilical 3 -cpm activity reflects that of the colon. Therefore, it is suggested that the acute stress of the MDT generally inhibited the gastric EGG and facilitated the colonic EGG, indicating gastric inhibition and colonic facilitation. The topographic EGG maps of the power content ratios and the absolute power ratios visually confirmed these findings.
\end{abstract}

Key words: electrogastrography (EGG), stress, mirror drawing test (MDT), topographic EGG map

\section{Introduction}

The facilitation or inhibition of the gastrointestinal tract by stress has been previously reported (Holtmann and Enck, 1991; Musial and Enck, 1993). In addition, the electromyographic activity of

Correspondence to: Shinji Homma, MD and PhD (Professor emeritus), Apt. 101, Igaku-kyoukai-shukusha, Hashimoto 920-1, Nishikan-ku, Niigata City 953-0103, Japan

e-mail: snjhomma@snow.plala.or.jp

C2012 The Japan Society of Smooth Muscle Research 
an EGG has been shown to partially reflect gastro-intestinal motility (Smout et al., 1980; Mintchef and Bowes, 1997). Therefore, it is possible to record the effects of stress on gastrointestinal activity using EGG (Homma, 2005, 2006, 2009). We have previously reported the facilitation or inhibition of gastrointestinal activity by recording the EGG at 16 locations on the thoraco-abdominal surface, by means of 1) the peak power ratio (peak power during MDT/peak power at rest) at locations in each of the epigastric (CHI), supraumbilical (CHII) and infraumbilical regions (CHIII) and of 2) the maximal power foci (MPFs) obtained from each of 16 locations (Homma, 2005). For the three locations, CHI was the mean of channels 3, 4, 5 and 6, while CHII was the mean of channels 7, 8, 9, and CHIII was the mean of channels 12, 13, and 14 (Homma, 2005). However, local differences in the EGG were found clearly at rest and during the postprandial state according to the power amplitude and the power content by numerically comparing the 16 channels (Homma, 2009). Therefore, in the present study, we tried to compare the effects of stress due to the MDT at each of the 16 locations using the ratio of the power content during MDT to that at rest (power content during MDT/power content at rest, \%/rest) and of the ratio of the absolute power during MDT to that under resting conditions (absolute power during MDT/ absolute power at rest, abs/r) to further define the local differences in the MDT stress responses on the EGG. The effects of a test meal were also compared by means of the same indices. The acute MDT stress inhibited the epigastric 3-cpm EGG (channels 5 and 8) and facilitated the infraumbilical 3-cpm EGG (channels 12-16). In contrast, eating a meal facilitated the epigastric 3-cpm EGG. Therefore, it appears that the acute stress of the MDT inhibited the gastric myoelectric activity and facilitated the colonic activity. Topographic EGG maps visually demonstrated these findings.

\section{Methods}

This project was conducted under the approval of the ethics committee of Niigata University, Faculty of Medicine (project no. 179). Informed consent was obtained from all of the healthy volunteers. Data were obtained during the course of a physiology laboratory. There were 58 subjects, 52 males and 6 females, ranging in age from $20-38$ years $(23.1 \pm 1.0$ for $n=23$, but the exact age of the remaining 35 subjects was not known). Postprandial sampling was performed after the subjects consumed a test meal (Cup Noodle, $363 \mathrm{kcal} / \mathrm{cup}$, Nissin, Japan) ( $\mathrm{n}=54)$.

The methods used for recording and analyzing EGGs were the same as in the previous studies (Homma et al., 1999: Homma, 2005). Briefly, unipolar EGGs were recorded from the 16 locations (channels, ch) from the thoraco-abdominal skin surface (Fig. 1), using a reference electrode on the right leg. The amplifier was a modified electroencephalographic $(\mathrm{EEG})$ amplifier, with time constant $=$ $5 \mathrm{sec}$, high cut $=0.5 \mathrm{~Hz}$, low cut $=-6 \mathrm{~dB} /$ oct and high cut $=-12 \mathrm{~dB} /$ oct (Biotop 6R 12-4, NEC-Sanei, Japan). After cleaning the skin with ethanol, electrode cream was applied to the disc electrodes for the EEG $($ diameter $=11 \mathrm{~mm})$. The electrodes were fixed on the skin with surgical tape. Resting EGGs were recorded for about $20 \mathrm{~min}$ in fasted subjects, with the EEG sampled every $128 \mathrm{sec}$ (1 file). After recording the resting control data, subjects were exposed to the stress of the MDT. The MDT involves using an electric pen to trace the cue image of a metal star reflected onto a mirror. The pen gives a click alarm whenever an error is made when the tracing runs off the edge of the star. The MDT stress was applied for about $5 \mathrm{~min}$ to obtain 2-3 EGG files. About $10 \mathrm{~min}$ after finishing the MDT test, a fur- 


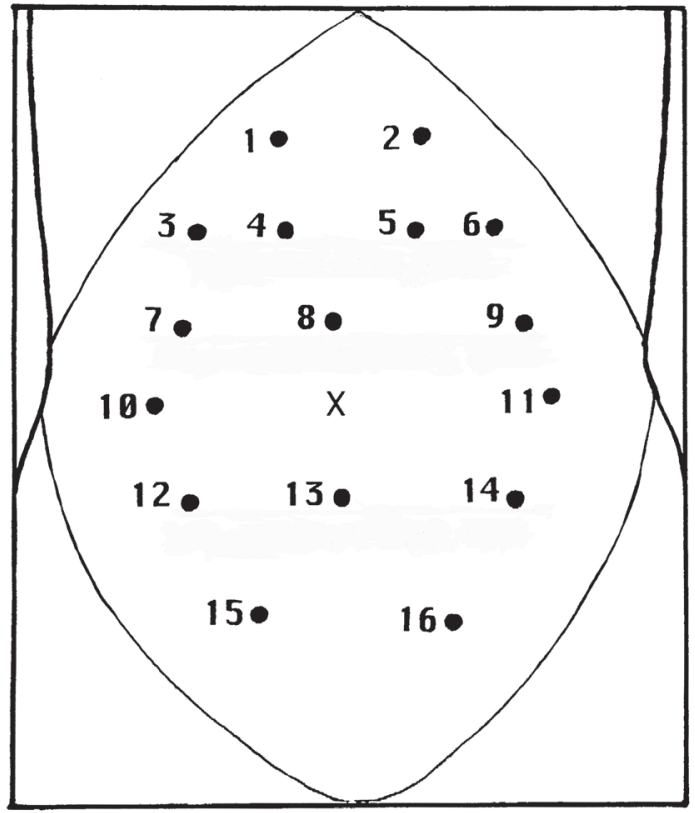

Fig. 1. Schematic presentation of the 16 locations of electrodes on the thoraco-abdominal surface $\mathrm{X}$ indicates the navel.

ther 20 min of EGG recordings (about 7 files) were collected to obtain a postprandial spectrum. The piled running spectra were obtained after the files were analyzed using the maximal entropy method (MEM). The spectral frequency readings were classified into five groups: the 1-cpm group (0-2.4 cpm), 3-cpm group (2.5-4.9 cpm), 6-cpm group (5.0-7.4 cpm), 8-cpm group (7.5-9.9 cpm) and 10-cpm group $(10.0-12.9 \mathrm{cpm})$. Ensemble means were obtained during rest, during the stress of the MDT, and after consuming a meal. With regard to the EGG parameters, this study focused on the power content ratio of each of the 16 channels, the MDT and postprandial power content ratio (power content during MDT/ power content at rest: MDT \%/r and power content after a meal/power content at rest: PPR \%/r). In addition, the absolute power ratio during MDT to rest (MDT abs/r) and postprandial state to rest (PPR abs/r) were similarly calculated. Tables 1 to 2 list the numerical figures of epigastric channels 5 and 8 and the infraumbilical channels 12-16 recorded in a previous study for simplicity (Homma, 2009). The statistical significances of the differences in epigastric and infraumbilical channels during MDT or the postprandial state are also shown in the tables, but those between MDT and the postprandial state were not reported in this study.

To make topographic EGG maps, the electrode positions were represented by two-dimensional standard coordinates, $\mathrm{Xi}$ and $\mathrm{Yi}$, and a spectral peak at a certain electrode position was expressed as $\mathrm{Zi}=(\mathrm{Xi}, \mathrm{Yi})$ (Homma, 1997: Homma et al., 1999: Homma, 2006). Thereafter, the contour map program (Shiono et al., 1988) was applied and EGG maps were drawn for the power content ratio (Homma, 1997). The iso-power content ratio lines and iso-absolute power ratio lines were usually drawn at intervals of $1 / 20$ of the maximal power content ratio and absolute power ratio of each spectral group to obtain the foci of the maximal power content ratio or maximal absolute power ratio as in the case of maximal power foci (MPFs) (Homma et al., 1999). 
The mean and standard errors (SE) were calculated and the Student's $t$-test was used to determine the statistical significance. $P$ values below 0.05 were considered to be significant.

\section{Results}

The comparisons of the resting and postprandial absolute power and absolute power during MDT data are show in Table 1 (1) to 1 (3). Similarly, comparisons of the postprandial absolute power ratio (abs/r) and in MDT absolute power ratio (abs/r) are shown in Table 1 (4) and Table 1 (5). In addition, comparisons of the power content ratio in the postprandial state $(\% / \mathrm{r})$, and during MDT $(\% / \mathrm{r})$ are shown in Table 2 (1) and 2 (2) (\%/r). Generally, during MDT stress, the absolute power, power ratio, and power content ratio of the epigastric channels 5 and 8 were lower than those of the infraumbilical channels $12-16$ for the 3 -cpm groups. In contrast, consuming a meal induced epigastric facilitation in the 3-cpm groups (Table 1). These tendencies are quite notable in the power content ratio $(\% / \mathrm{r})$, with significant differences found between the epigastric and infraumbilical channels in the 3-cpm group (Table 2).

Topographic EGG maps of the absolute power in the resting (f) and post-prandial states (ppr) and during MDT (MDT) are shown in Fig. 2. Intense foci were found in the 3-cpm group in the infraumbilical area during MDT (Fig. 2, 3rd row) and in the epigastric area in the postprandial state (Fig. 2, 2nd row). These findings are clearly seen in the topographic EGG maps based on the power ratios. A focus of red and higher power ratios of the 3-cpm group is seen in the epigastric area in the postprandial state and in the infraumbilical area during MDT (Fig. 2, $4^{\text {th }}$ and $5^{\text {th }}$ rows). These tendencies are most clearly seen in topographic EGG maps of the power content ratios (Fig. 3). The power content ratios in the epigastric area are low with predominantly yellow to orange areas, while the infraumbilical region had high power content ratios with predominantly purple to red areas during MDT. In contrast, the epigastric regions showed high power content ratios, with predominantly purple to red areas in the postprandial state.

\section{Discussion}

It is well known that stress influences gastric, small intestinal and colonic activity. In fact, stress induces dual excitatory and inhibitory effects on gastrointestinal activity (Holtmann and Enck, 1991: Musial and Enck, 1993). We have previously reported the effects of acute stress of MDT on the gastric and colonic facilitation and inhibition with EGG (Homma, 2005, 2006, 2009).

\section{Intestinal activity}

No significant difference in the power content ratios were found in either the 8 or $10 \mathrm{cpm}$ groups during MDT (Table 1). In addition, it is not clear whether the supraumbilical and infraumbilical higher power content ratio areas in topographic EGG maps of the power content ratio reflect colonic or intestinal facilitation during MDT. It has been reported that colonic activity includes a wide range of spectral frequency activity in the 3 to 10 -cpm range, including intestinal activity (Sarna, 1989). It is possible 
Table 1. Comparison of the absolute power in the fasting state Table 1 (1), (f) $\left(x 1,000 \mu V^{2}\right)(n=58)$, postprandial state Table $1(2),(\mathrm{ppr})\left(\mathrm{x} 1,000 \mu \mathrm{V}^{2}\right)(\mathrm{n}=53-55)$ and, during MDT, Table 1 (3) $\left(\mathrm{x} 1,000 \mu \mathrm{V}^{2}\right),(\mathrm{n}=53-58)$. Absolute power ratio, $\mathrm{ppr} / \mathrm{r}$, abs/r, Table 1 (4) and absolute power ratio, MDT/rest, abs/r, Table 1 (5).

Table 1 (1). fasting (f)

\begin{tabular}{llcccc}
\hline Fasting & 1-cpm (0-2.4) & 3-cpm (2.5-4.9) & 6-cpm (5.0-7.4) & 8-cpm (7.5-9.9) & 10-cpm (10.0-12.9) \\
\hline ch. 5f & $25.1 \pm 4.14$ & $53.8 \pm 7.81$ & $15.0 \pm 3.37$ & $10.0 \pm 2.36$ & $8.73 \pm 1.24$ \\
ch. 8f & $35.9 \pm 7.51$ & $57.3 \pm 15.5$ & $14.5 \pm 2.61$ & $14.2 \pm 2.40$ & $20.2 \pm 5.00$ \\
ch.12f & $46.0 \pm 15.9$ & $20.9 \pm 7.08 \mathrm{~b}$ & $34.9 \pm 15.0$ & $15.6 \pm 4.49$ & $9.65 \pm 1.68$ \\
ch.13f & $27.6 \pm 5.52$ & $16.3 \pm 3.07 \mathrm{a}, \mathrm{z}$ & $9.41 \pm 2.19$ & $12.0 \pm 3.03$ & $14.8 \pm 2.93$ \\
ch.14f & $40.9 \pm 7.96$ & $20.1 \pm 3.47 \mathrm{~b}, \mathrm{z}$ & $11.1 \pm 2.26$ & $13.9 \pm 4.07$ & $10.2 \pm 1.52$ \\
ch.15f & $32.5 \pm 10.2$ & $17.2 \pm 5.67 \mathrm{~b}, \mathrm{z}$ & $11.8 \pm 3.65$ & $9.81 \pm 2.70$ & $6.43 \pm 1.44 \mathrm{z}$ \\
ch.16f & $26.3 \pm 4.80$ & $12.7 \pm 1.90 \mathrm{a}, \mathrm{z}$ & $7.71 \pm 1.43 \mathrm{z}$ & $9.77 \pm 2.92$ & $8.02 \pm 1.18 \mathrm{z}$ \\
\hline
\end{tabular}

The significant differences between channel 5 and the other channels are indicated by $\mathrm{a}, \mathrm{b}$, and $\mathrm{c}$ and between channel 8 and the others by $\mathrm{x}, \mathrm{y}$, and $\mathrm{z}$, with. a and $\mathrm{x} P<0.001, \mathrm{~b}$ and $\mathrm{y} P<0.01, \mathrm{c}$ and $\mathrm{z}, P<0.05$. Between $\mathrm{p}$ and $\mathrm{q}, P<0.05$; between $\mathrm{r}$ and $\mathrm{s}, P<0.01$.

Table 1 (2). postprandial (p)

\begin{tabular}{cccccc}
\hline \multicolumn{1}{c}{$\mathrm{p}$} & 1-cpm (0-2.4) & 3-cpm (2.5-4.9) & 6-cpm (5.0-7.4) & 8-cpm (7.5-9.9) & 10-cpm (10.0-12.9) \\
\hline ch.5p & $54.0 \pm 10.1$ & $92.6 \pm 13.2$ & $21.2 \pm 3.94$ & $14.5 \pm 2.36$ & $10.6 \pm 1.20$ \\
ch.8p & $50.5 \pm 7.41$ & $94.8 \pm 20.9$ & $31.8 \pm 7.54$ & $36.4 \pm 12.3$ & $16.0 \pm 1.91$ \\
ch.12p & $73.3 \pm 23.0$ & $31.5 \pm 7.56 \mathrm{~b}, \mathrm{z}$ & $26.6 \pm 7.02$ & $22.3 \pm 6.05$ & $7.96 \pm 0.94$ y \\
ch.13p & $61.1 \pm 12.7$ & $39.1 \pm 12.2 \mathrm{~b}, \mathrm{z}$ & $15.3 \pm 2.90$ & $16.0 \pm 3.21$ & $11.1 \pm 1.55$ \\
ch.14p & $76.9 \pm 19.6$ & $39.3 \pm 8.76 \mathrm{~b}, \mathrm{z}$ & $24.1 \pm 5.26$ & $19.7 \pm 4.78$ & $10.1 \pm 1.33$ \\
ch.15p & $69.3 \pm 19.8$ & $41.1 \pm 10.8 \mathrm{~b}, \mathrm{z}$ & $17.5 \pm 3.32$ & $11.1 \pm 2.50$ & $6.50 \pm 1.04 \mathrm{c}, \mathrm{z}$ \\
ch.16p & $67.8 \pm 15.8$ & $37.2 \pm 9.18 \mathrm{c}, \mathrm{z}$ & $16.2 \pm 2.76$ & $14.6 \pm 4.36$ & $17.0 \pm 8.37$ \\
\hline
\end{tabular}

The significant differences between channel 5 and the other channels are indicated by $\mathrm{a}, \mathrm{b}$, and $\mathrm{c}$ and between channel 8 and the others are indicated by $\mathrm{x}, \mathrm{y}$, and $\mathrm{z}$. a and $\mathrm{x}, P<0.001 ; \mathrm{b}$ and $\mathrm{y}, P<0.01$; $\mathrm{c}$ and $\mathrm{z}, P<0.05$. Between $\mathrm{p}$ and $\mathrm{q}, P<0.05$; between $\mathrm{r}$ and $\mathrm{s}, P<0.01$.

Table 1 (3). Mirror Drawing Test (MDT)

\begin{tabular}{lccccc}
\hline MDT & 1-cpm (0-2.4) & 3-cpm (2.5-4.9) & 6-cpm (5.0-7.4) & 8-cpm (7.5-9.9) & 10-cpm (10.0-12.9) \\
\hline ch.5L & $316 \pm 55$ & $150 \pm 29$ & $89 \pm 23$ & $62 \pm 15$ & $30 \pm 6.7$ \\
ch.8L & $284 \pm 49$ & $182 \pm 35$ & $114 \pm 35$ & $55 \pm 14$ & $35 \pm 6.5$ \\
ch.12L & $392 \pm 74$ & $173 \pm 38$ & $124 \pm 40$ & $58 \pm 15$ & $29 \pm 6.2$ \\
ch.13L & $341 \pm 66$ & $196 \pm 54$ & $93 \pm 22$ & $51 \pm 13$ & $33 \pm 6.8$ \\
ch.14L & $504 \pm 145$ & $196 \pm 49$ & $116 \pm 30$ & $48 \pm 11$ & $39 \pm 7.8$ \\
ch.15L & $327 \pm 60$ & $180 \pm 40$ & $102 \pm 26$ & $50 \pm 14$ & $31 \pm 7.4$ \\
ch.16L & $336 \pm 60$ & $159 \pm 37$ & $88 \pm 23$ & $56 \pm 14$ & $34 \pm 6.1$ \\
\hline
\end{tabular}

Table 1 (4). The effect of a test meal effect on absolute power to rest ( $\mathrm{ppr} / \mathrm{r}, \mathrm{abs} / \mathrm{r}, \mathrm{n}=53-55)$.

\begin{tabular}{llcccc}
\hline & 1-cpm (0-2.4) & 3-cpm (2.5-4.9) & 6-cpm (5.0-7.4) & 8-cpm (7.5-9.9) & 10-cpm (10.0-12.9) \\
\hline ch.5 & $2.87 \pm 0.64$ & $5.51 \pm 1.56$ & $1.02 \pm 0.21$ & $0.93 \pm 0.27$ & $0.64 \pm 0.12$ \\
ch.8 & $2.31 \pm 0.41$ & $3.60 \pm 1.10$ & $1.34 \pm 0.32$ & $1.43 \pm 0.37$ & $1.07 \pm 0.25_{1}$ \\
ch.12 & $3.41 \pm 0.72$ & $2.26 \pm 0.86$ & $1.27 \pm 0.33$ & $1.20 \pm 0.45$ & $0.46 \pm 0.009_{2}$ \\
ch.13 & $3.00 \pm 0.83$ & $2.16 \pm 0.97$ & $0.95 \pm 0.31$ & $0.94 \pm 0.26$ & $0.64 \pm 0.13$ \\
ch.14 & $3.46 \pm 0.74$ & $3.00 \pm 1.48$ & $1.20 \pm 0.28$ & $1.05 \pm 0.31$ & $0.61 \pm 0.13$ \\
ch.15 & $2.51 \pm 0.56$ & $2.67 \pm 1.20$ & $1.01 \pm 0.27$ & $0.67 \pm 0.18$ & $0.36 \pm 0.008_{3}$ \\
ch.16 & $2.95 \pm 0.59$ & $2.66 \pm 1.10$ & $0.98 \pm 0.25$ & $0.77 \pm 0.18$ & $0.61 \pm 0.16$ \\
\hline
\end{tabular}

Statistical significance: between $1-2$, and $1-3, P<0.05$.

Tabl 1 (5). The effect of the MDT on absolute power to rest (MDT/r, abs/r, n=52-58).

\begin{tabular}{lllccc}
\hline & 1-cpm (0-2.4) & 3-cpm (2.5-4.9) & 6-cpm (5.0-7.4) & 8-cpm (7.5-9.9) & 10-cpm (10.0-12.9) \\
\hline ch.5 & $24.2 \pm 6.3$ & $12.1 \pm 5.0$ & $11.9 \pm 4.1$ & $11.2 \pm 3.6$ & $4.4 \pm 1.1$ \\
ch.8 & $19.6 \pm 4.3$ & $14.5 \pm 6.0$ & $36.7 \pm 25.0$ & $8.1 \pm 2.6$ & $3.7 \pm 1.01$ \\
ch.12 & $28.2 \pm 7.8$ & $30.4 \pm 10.2$ & $21.6 \pm 8.6$ & $11.3 \pm 4.5$ & $4.9 \pm 1.2$ \\
ch.13 & $25.1 \pm 6.1$ & $40.3 \pm 20.6$ & $20.7 \pm 5.2$ & $10.5 \pm 3.6$ & $4.3 \pm 0.9$ \\
ch.14 & $40.4 \pm 14.5$ & $24.3 \pm 8.5$ & $17.8 \pm 5.6$ & $13.9 \pm 4.6$ & $8.5 \pm 2.9$ \\
ch.15 & $26.2 \pm 6.2$ & $34.0 \pm 10.0$ & $22.9 \pm 6.3$ & $12.7 \pm 4.4$ & $9.2 \pm 2.3_{2}$ \\
ch.16 & $24.6 \pm 5.4$ & $33.4 \pm 12.9$ & $19.9 \pm 5.4$ & $18.1 \pm 6.0$ & $7.9 \pm 2.0$ \\
\hline
\end{tabular}

Statistical significance, between 1 and $2, P<0.05$. 
Table 2 (1). The effects of consuming a test meal effect on the power content ratio $(\% / r, n=52-55)$.

\begin{tabular}{llcccc}
\hline & 1-cpm (0-2.4) & 3-cpm (2.5-4.9) & 6 -cpm (5.0-7.4) & 8 -cpm (7.5-9.9) & 10-cpm (10.0-12.9) \\
\hline ch.5 & $0.88 \pm 0.072_{1}$ & $1.18 \pm 0.077_{8}$ & $0.50 \pm 0.040$ & $0.37 \pm 0.050$ & $0.30 \pm 0.027$ \\
ch.8 & $0.83 \pm 0.061_{2}$ & $1.01 \pm 0.061_{9}$ & $0.54 \pm 0.053$ & $0.47 \pm 0.062$ & $0.37 \pm 0.041_{16}$ \\
ch.12 & $1.15 \pm 0.100_{3}$ & $0.81 \pm 0.062_{10}$ & $0.55 \pm 0.061$ & $0.42 \pm 0.055$ & $0.32 \pm 0.096$ \\
ch.13 & $1.06 \pm 0.083_{4}$ & $0.86 \pm 0.067_{11}$ & $0.50 \pm 0.040$ & $0.44 \pm 0.044$ & $0.35 \pm 0.031_{17}$ \\
ch.14 & $1.10 \pm 0.089_{5}$ & $0.86 \pm 0.063_{12}$ & $0.58 \pm 0.057$ & $0.43 \pm 0.049$ & $0.33 \pm 0.02718$ \\
ch.15 & $1.18 \pm 0.100_{6}$ & $0.96 \pm 0.085$ & $0.54 \pm 0.054$ & $0.37 \pm 0.037$ & $0.24 \pm 0.019$ \\
ch.16 & $1.12 \pm 0.085_{7}$ & $0.90 \pm 0.070_{13}$ & $0.52 \pm 0.039$ & $0.40 \pm 0.033$ & $0.30 \pm 0.027$ \\
\hline
\end{tabular}

Statistical significances: $1-3,1-6,1-7,2-3,2-4,2-5,2-7,8-15,18-19, P<0.05 .2-6,8-10,8-11,8-12,9-10$, $16-19$ and 17-19: $P<0.01$.

Table 2 (2). The effect of MDT on the power content ratio $(\% / r, n=52-58)$.

\begin{tabular}{|c|c|c|c|c|c|}
\hline & $1-\mathrm{cpm}(0-2.4)$ & $3-\operatorname{cpm}(2.5-4.9)$ & $6-\operatorname{cpm}(5.0-7.4)$ & 8-cpm (7.5-9.9) & $10-\operatorname{cpm}(10.0-12.9)$ \\
\hline ch.5 & $1.95 \pm 0.26$ & $0.90 \pm 0.054_{4}$ & $0.89 \pm 0.060$ & $0.96 \pm 0.089$ & $0.84 \pm 0.10$ \\
\hline ch. 8 & $1.71 \pm 0.16$ & $1.01 \pm 0.066$ & $1.08 \pm 0.10$ & $0.84 \pm 0.073$ & $0.83 \pm 0.096$ \\
\hline ch.12 & $1.64 \pm 0.17$ & $1.22 \pm 0.090_{5}$ & $0.96 \pm 0.074$ & $0.82 \pm 0.088$ & $0.67 \pm 0.096$ \\
\hline ch.13 & $1.63 \pm 0.19$ & $1.21 \pm 0.074$ & $1.03 \pm 0.084$ & $0.74 \pm 0.076$ & $0.66 \pm 0.081$ \\
\hline ch.14 & $1.48 \pm 0.13$ & $1.30 \pm 0.12$ & $0.99 \pm 0.081$ & $0.84 \pm 0.12$ & $1.28 \pm 0.57$ \\
\hline ch. 15 & $1.32 \pm 0.11_{2}$ & $1.17 \pm 0.076_{8}$ & $1.04 \pm 0.073$ & $0.87 \pm 0.10$ & $0.79 \pm 0.11$ \\
\hline ch.16 & $1.34 \pm 0.11$ & $1.19 \pm 0.092$ & $1.04 \pm 0.077$ & $0.81 \pm 0.073$ & $0.69 \pm 0.070$ \\
\hline
\end{tabular}

Statistical significances: $1-2$ and $1-3, P<0.05 .4-5,4-6,4-7$ and $4-8, P<0.01 .4-9, P<0.05$.

that the postprandial supraumbilical facilitation seen in the topographic EGG maps might be indicative of postprandial upper intestinal facilitation.

With regard to the effects of stress on intestinal activity, dichotic listening, video games and mental stress all decreased intestinal motility, as observed by the number of myoelectric motor complexes (MMCs) measured by manometry (McRae et al., 1982: Valori et al., 1986; Holtmann et al., 1989), but increased in the transit time in the human jejunum (Barcley and Turnberg, 1987).

\section{Colonic activity}

Both colonic facilitation and inhibition were suggested by MPFs with isopower topographic EGG maps (Homma, 2005). A significantly higher power content ratio in the infraumbilical 3-cpm range than in that of the epigastric area during MDT with EGG (Table 2) suggests that MDT stress inhibited the gastric EGG and facilitated the colonic EGG. The findings from the topographic EGG maps drawn according to the power content ratios and the absolute power ratios during MDT stress further support this conclusion. The yellow epigastric lower power content area and the red to purple infraumbilical higher power content area (Fig. 3, 2nd row) visually confirmed this suggestion. It is possible that postprandial infraumbilical facilitation in the 1-cpm groups might reflect the gastro-colic reflex (Homma, 2009).

Similar findings have been reported with manometry. For example, MDT-stress induced a significant increase in the colonic motility of patients with irritable bowel syndrome, with an increase in heart rate variability, but had no effect in healthy controls (Fukudo and Suzuki, 1987). Cold presser test stress, interviews and arithmetic calculations increased colonic motility as measured by both manometry and EGG (Chaudhary and Truelove, 1961; Nruducci et al., 1985; Rao et al., 1998; Welgan et al., 1985). Psychological stressors producing anger and anxiety also increased the colonic motility in normal healthy controls and irritable bowel syndrome patients (Welgan et al., 1985, 1988). MDT-related 

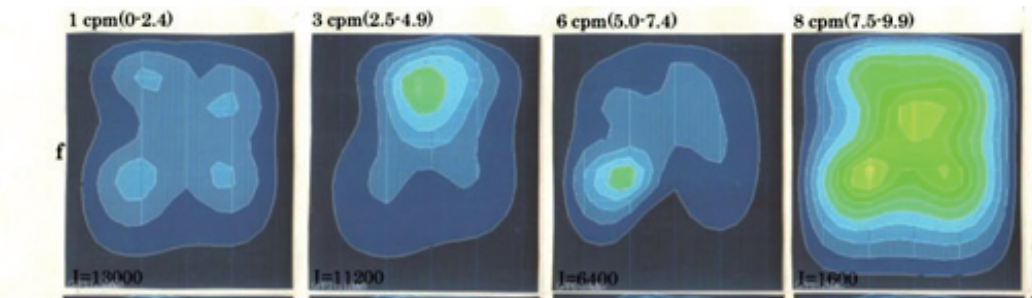

$10 \mathrm{cpm}\left(10.0^{-12 .)}\right.$
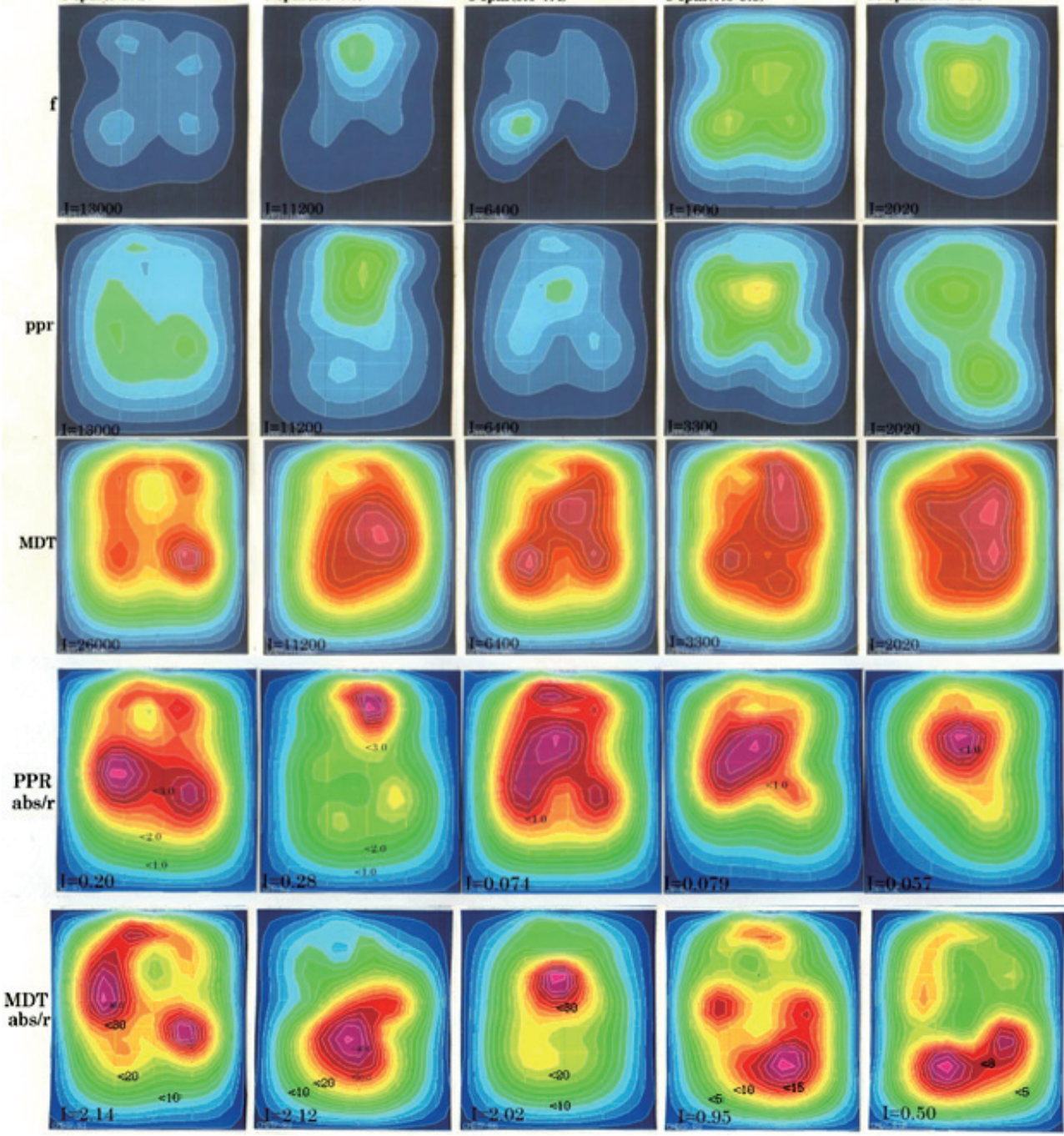

Fig. 2. Table 1 (3) shows the epigastric inhibition and infraumbilical facilitation during MDT in the 3 cpm group in a comparison of the absolute power. In contrast, Table 1 (2) shows epigastric facilitation postprandially in the 3-cpm group. Accordingly, topographic EGG maps visually demonstrated these findings. Topographic EGG maps are shown during rest (f), after having a test meal (ppr), and during MDT based on absolute power alone (Fig. 2, first to third row). Intervals of the iso-power line were 13,000 micro $\mathrm{V}^{2}$ for 1-cpm of (f) and (ppr), and 26,000 for the 1-cpm group of (MDT). 11,200 for all the 3-cpm groups of (f), (ppr), and (MDT), 6,400 for all 6-cpm groups, 1,600 for the 8-cpm groups of (f), 3,300 for the 8-cpm groups of both (ppr) and (MDT) and 2,020 for all 10-cpm groups as shown in the figures. Absolute power data are shown in Table 1. Table 1 (1-2) have already been presented in the previous paper (Homma, 2009). Table 1 (3) is added in this paper. The epigastric maximal power focus in the 3-cpm group is in the epigastrium during the resting state (f). In contrast, the maximal power focus during MDT is on the left side of the navel, not in the epigastrium. The powers are shown from higher to lower as purple, red, orange, yellow, green to blue. Absolute power ratios of abs/r (for both ppr/r and MDT/r) are shown in Tables 1 (4) and 1 (5) and similar results for absolute power ratios are found in the topographic EGG of $\mathrm{ppr} / \mathrm{r}$ (4th row) and MDT/r ( $5^{\text {th }}$ row). Intervals for the power ratios are also $1 / 20$ of the maximal power ratio for both $\mathrm{ppr} / \mathrm{r}$ and MDT/r. Accordingly, even the topographic EGG maps based on absolute power itself and the absolute power ratios demonstrated gastric inhibition and colonic facilitation during MDT and postprandial epigastric facilitation. Approximate power ratios are indicated by symbol $(<)$ and numerical figures, such as $<1.0,2.0$, or $<5,8,10,20$, and 30 . 

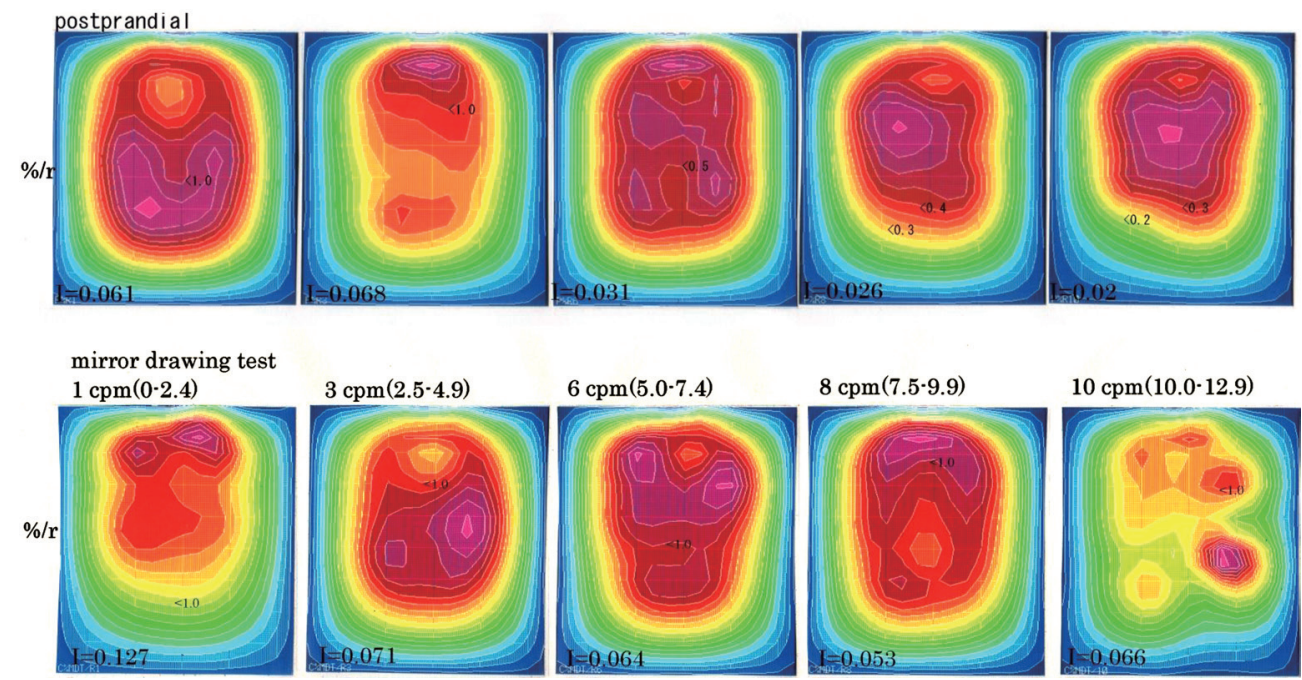

Fig. 3. Topographic EGG maps 1) after a meal (\%/r, power content after having a meal/power content at rest) (first row), and 2) during MDT (\%/r, power content during MDT/power content at rest) (2nd row). The ratios are shown from higher to lower ratios as purple, red, orange, yellow, green to blue. The actual ratios are shown by arrows $(<)$ and numerical figures, such as $<0.1$ to 0.5 , and $<1.0$. In the second row of the 3 -cpm group, the epigastric yellow to orange area and infraumbilical purple to red area indicate lower epigastric and higher infraumbilical power content ratios compared to those seen in Table 2. Conversely, in the first row 3-cpm group, the epigastric purple to red and infraumbilical orange to yellow colors indicate the higher epigastric and lower infraumbilical power content ratio as seen in Table 2. The letter I in the left lower edge of each figure indicates the interval of the iso-power content ratios, or iso-power ratios (usually as $1 / 20$ of the maximal power content ratio).

stress significantly increased the bowel frequency, while depressed patients tended to be constipated (Gorard et al., 1996).

\section{Gastric activity}

There was a significantly lower power content ratio in the epigastric area of the 3-cpm group compared to that of the infraumbilical area, suggesting that there is a gastric EGG inhibition during MDT (Table 2 (2)). Conversely, the postprandial power content ratio of the epigastric area of the 3-cpm group was significantly higher than that of the infraumbilical area (Table 2 (1)). This finding is further supported by the topographic EGG maps of the postprandial power content ratios according to the epigastric red to purple higher power content ratio area after a meal (Fig. 3, 1st row).

The postprandial power increases in the 3-cpm component is well known (Smout et al., 1980). EGG is a non-invasive and non-stressful method for studying the gastrointestinal myoelectric activity, which reflects some of the motility (Smout et al., 1980: Mintchef and Bowes, 1997). Therefore, our data, combined with that in previous studies, suggests that MDT stress inhibited gastric activity and facilitated colonic activity.

Similar gastric inhibitions or facilitation by stress have been reported in other studies. For example, noise increased the amplitude of EGGs (Fedor and Russel, 1965), while the cold stress test decreased gastric motility as measured manometrically (Fone et al., 1990). The arithmetic task stress induces a significant increase in the dominant gastric power and a slight decrease in the EGG dominant 
gastric frequency in healthy subjects (Riezzo et al., 1996). Electrical shock significantly decreased the percentage in the 3-cpm frequency group and tachyarrhythmia (\%) component of the EGG, but forehead cooling increased the percentage of the 3-cpm frequency group (Muth et al., 1999). Anxiety has also been reported to facilitate antral meal retention (Lorena et al., 2004).

The epigastric 3- and 6-cpm groups electrical activity in the EGG were suggested to reflect those of the colon on the basis of the EGG results obtained after total gastrectomy (Pezzola et al., 1989; Homma et al., 1995, 1999). We have reported that gastric, intestinal and colonic myoelectric activity can be recorded using EGG (Homma et al., 1995: Homma, 1997: Homma et al., 1999, 2000). Topographical EGG maps were also drawn for the determination of maximal power foci (MPFs) in the resting state, during MDT and after consuming a meal (Homma, 1997: Homma et al., 1999, 2000: Zhou et al., 1997). It appears that the infraumbilical $3 \mathrm{cpm}$ EGG activity might reflect colonic electrical activity (Pezzola et al., 1989; Sarna et al., 1989; Homma et al., 1995, 1999; Amaris et al., 2002). EGG records the gastrointestinal electrical activity or myoelectric activity, which reflects some of the motility (Smout et al., 1980: Mintchef and Bowes, 1997). The various stressors depress the stomach contractility and emptying, and facilitate the colonic motility, transit and defecation via 1) CRF-R2 (corticotrophinreleasing factor receptor 2) and CRF-R1, respectively (Monniles et al., 1992; Tache et al., 1993; Tache et al., 2001: Bhatia and Tandon, 2005). Our present EGG findings in human subjects provide further support for these studies.

Finally, the topographic EGG maps drawn from the power content ratios and the absolute power ratios at each electrode location may accurately reflect physiological topographic changes due to MDT or postprandial effects than those drawn from either the power content or absolute power alone.

\section{References}

Amaris, M.A., Sanmiguel, C.P., Sadowski, D.C., Bowes, K.L. and Mintchef, M.P. (2002). Electrical activity from colon overlaps with normal gastric electrical activity in cutaneous recordings. Dig. Dis. Sci. 47: 2480-2485.

Barcley, G.R. and Thurnberg, L.A. (1987). Effects of psychological stress on salt and water transport in the human jejunum. Gastroenterol. 93: 91-97.

Bhatia, V. and Tandon, R.K. (2005). Stress and the gastrointestinal tract. J. Gastroenterol. Hepatol. 20: 332-33.

Chaudhary, Y.N.A. and Truelove, S.C. (1961). Human colonic motility: a comparable study of normal subjects, patients with ulcerative colitis, and patients with the irritable colon syndrome. I. Resting patterns of motility. Gastroenterol. 40: 1-17.

Fedor, J.H. and Russel, R.W. (1965). Gastrointestinal reactions to response-contingent stimulation. Psychol. Rev. 16: 95-111.

Fone, D.R., Horowitz, M., Maddox, A., Akkermans, L.M., Read, N.W. and Dent, J. (1990). Gastrointestinal motility during the delayed gastric emptying induced by cold stress. Gastroenterol. 98: 1155-1161.

Fukudo, S. and Suzuki, J. (1987). Colonic motility, autonomic function, and gastrointestinal hormones under psychological stress on irritable bowel syndrome. Tohoku J. Exp. Med. 151: 373-385.

Gorard, D.A., Gomborone, J.F., Libby, G.W. and Farthing, M.J. (1996). Intestinal transit in anxiety and depression. Gut 39: 551-555.

Holtmann, G. and Enck, P. (1989). Differential effects of acute mental stress on gastric acid, pancreatic enzyme secretion and gastroduodenal motility. Dig. Dis. Sci. 34: 1701-1707.

Holtmann, G. and Enck, P. (1991). Stress and gastrointestinal motility in humans: A review of the literature. $J$. 
Gastrointest. Mot. 3: 245-254.

Homma, S. (1997). Isopower mapping of the electro-gastrogram (EGG). J. Auton. Nerv. Sys. 62: 163-166.

Homma, S. (2005). The effects of stress in response to mirror drawing test trials on the electrogastrogram, heart rate and respiratory rate of human subjects. J. Smooth Muscle Res. 41: 221-233.

Homma, S. (2006). Correlations between the responses of electrogastrograms, heart rate and respiratory rate to the stress of the mirror drawing test in human subjects. J. Smooth Muscle Res. 42: 9-19.

Homma, S. (2009). Local differences in electrogastrograms recorded on thoraco-abdominal surface at 16 locations. J. Smooth Muscle Res. 45: 299-306.

Homma, S., Hasegawa, J., Maruta, T., Watanabe, N., Matsuo, H., Tamiya, Y., Nishimaki, T., Suzuki, T., Muto, T. and Hatakeyama, K. (1999). Isopower maps of the electrogastrogram (EGG) after total gastrectomy or total colectomy. Neurogastroenterol. Motil. 11: 441-448.

Homma, S. Shimakage, N., Yagi, M., Hasegawa, J., Satoh, K., Matsuo, H., Tamiya, Y., Tabnaka, O., Muto, T. and Hatakeyama, K. (1995). Electrogastrography prior to and following total gastrectomy, subtotal gastrectomy, and gastric tube formation. Dig. Dis. Sci. 40: 893-900.

Homma, S., Yagi, M., Uciyama, M., and Iwafuchi, M. (2000). Isopower mapping of electrogastrograms in short bowel syndrome. Med. Biol. Eng. Comp. 38: 653-658.

Lorena, S.L., Tinois, E., Brunetto, S.Q., Camargo, E.E. and Mesquita, M.A. (2004). Gastric emptying and intragastric distribution of a solid meal in functional dyspepsia: influence of gender and anxiety. $J$. Clin. Gastroenterol. 38: 230-236.

McRae, S., Younger, K., Thompson, D.G. and Wingate, D.K. (1982). Sustained mental stress alters human jejunal motor activity. Gut 23: 230-236.

Mintchev, M.P. and Bowes, K.L. (1997). Do increased electrogastrographic frequencies always correspond to internal tachygastria? Ann. Biomed. Eng. 25: 1052-1058.

Monnikes, H., Schmidt, D.G., Raybould, H.E. and Tache, Y. (1992). CRF in the paraventricular nucleus mediate gastric and colonic motor response to restraint stress. Am. J. Physiol. 262: G137-G143.

Musial, F. and Enck, P. (1993). Stress and gastrointestinal motility. In: An illustrated Guide to gastrointestinal motility. $2^{\text {nd }}$ ed., Eds. by D. Kummar and D. Wingate, Churchill Hills, Livingstone, Edingburh, pp. $104-117$.

Muth, E.R., Koch, K.L., Stern, R.M. and Thayer, J.F. (1999). Effects of autonomic nervous system manipulation on gastric myoelectric activity and emotional responses in healthy human subjects. Psychosomatic Med. 61: 297-303.

Naruducci, R., Snape, W.J., Battle, W.M., London, R.L. and Cohen, S. (1985). Increased colonic motility during exposure to a stressful situation. Dig. Dis. Sci. 30: 40-44.

Pezzola, F., Riezzo, G., Maselli, M.A. and Giorgio, I. (1989). Electrical activity recorded from abdominal surface after gastrectomy or colectomy. Gastroenterol. 97: 313-320.

Rao, S.S.C., Hatfield, R.A., Suls, J.M. and Chamberlain, M.J. (1998). Psychological and physical stress induce differential effects on human colonic motility. Am. J. Gastroenterol. 93: 985-990.

Riezzo, G., Porcelli, P., Guerra, V. and Giorgio, I. (1996). Effects of different psychophysiological stressors on the cutaneous electrogastrogram in healthy subjects. Arch. Physiol. Biochem. 104: 282-286.

Sarna, S.K. (1989). Colonic electrical activity as an indicator of colonic motor function. In: Read,N.W. ed., Gastrointestinal Motility: What Test?; Petersfield, UK: Wrighston Biomedical Publishers, pp. 203-211.

Shiono, S., Masumoto, S. and Wadatsumi, K. (1988). Contour map by BASIC. II. (in Japanese): KyorituShuppan Co. Ltd., Tokyo, Japan, pp. 71-75.

Smout, A.J.P.M., Jebbink, H.J.A. and Samsom, M. (1994). Acquisition and analysis of electrogastrographic data. The Dutch experience. In: Electrogastrography. Principles and Applications., ed. by J.Z. Chen and R.W. McCallum, Raven Press, New York, pp. 3-30.

Smout, A.J.P.M. and van der Schee, E.T. (1989). Electrogastrography. In: Gastrointestinal motility. Which test?, ed. By N.W. Read, Wrightson Biomedical Publishing LTD, Petersfield, pp. 113-120.

Smout, A.J.P.M., van der Schee, E.T. and Grashuis, J.L. (1980). What is measured in electrogastrography? Dig. 
Dis. Sci. 25: 179-187.

Tache, Y., Martinez, V., Million, M. and Wang, L. (2001). Stress and the gastrointestinal tract III. Stressrelated alterations of gut motor functions: role of brain corticotropin-releasing factor receptors. $\mathrm{Am}$. J. Physiol. 280: G173-G177.

Tache, Y., Monnikes, H., Bonaz, B. and Rivier, J. (1993). Role of CRF in stress-related alteration of gastric and colonic motor function. Ann. N. Y. Acad. Sci. 697: 233-243.

Valori, R.M., Kumar, D. and Wingate, D.L. (1986). Effects of different types of stress and of prokinetic drugs on the control of the fasting motor complex in humans. Gastroenterol. 90: 1890-1900.

Welgan, P., Meshkinpour, H. and Beeler, M. (1988). Effect of anger on colonic motor and myoelectric activity in irritable bowel syndrome. Gastroenterol. 94: 1050-1056.

Welgan, P., Meshkinpour, H. and Hoehler, F. (1985). The effects of stress on motor and electrical activity in irritable bowel syndrome. Psychosom. Med. 47: 139-149.

Zhou, M., Zhang, H., Shaw, R. and Barnes, F.S. (1997). Real time multi-channel computerized electrogastrograph. IEEE Trans. BME. 44: 1228-1236. 